\title{
Developments in surface magneto-optical Kerr effect setup for ultrahigh vacuum analysis of magnetic ultrathin films
}

\author{
V. Usov, S. Murphy, L. Seravalli, and I. V. Shvets \\ SFI Nanoscience Laboratory, Department of Physics, Trinity College, Dublin 2, Ireland
}

(Received 21 October 2004; accepted 24 January 2005; published online 16 March 2005)

\begin{abstract}
We describe a surface magneto-optical Kerr effect (SMOKE) setup designed to study the magnetic properties of ultrathin films in ultrahigh vacuum (UHV). As in earlier systems, a crossed-poles electromagnet is used, which allows measurements in the longitudinal or polar configurations to be carried out without displacing the sample or changing the optical path. The poles are mounted in-vacuum, while the coils and yoke are mounted externally to obtain a higher magnetic field $(\sim 2.8 \mathrm{kOe})$. The width of the gap between the poles can be adjusted depending on the size of the sample. The performance of the instrument is demonstrated by measurements of the paramagnetic-ferromagnetic phase transition in a $2 \mathrm{ML} \mathrm{Fe}$ film grown on the Mo(110) surface. (C) 2005 American Institute of Physics. [DOI: 10.1063/1.1878192]
\end{abstract}

Epitaxial ultrathin films exhibit magnetic properties due to the reduced dimensionality, the presence of the mismatchinduced strain and the interaction with the substrate. There is also a strong correlation between the film morphology and properties such as the Curie temperature $T_{c}$ and the easy axis of magnetization. The surface magneto-optical Kerr effect (SMOKE) is a powerful method for probing the magnetic properties of these ultrathin film systems. ${ }^{1}$ When combined with a high-resolution imaging technique such as scanning tunneling microscopy (STM), it can be used to investigate the relationship between film morphology and magnetic properties under different growth conditions. In this article, we present an ultrahigh vacuum (UHV) compatible SMOKE system based on two pairs of crossed electromagnet poles that allow measurements to be performed in both the polar and longitudinal Kerr configurations. ${ }^{2}$ The UHV compatible design eliminates the need for a passivating layer, which in the case of ultrathin film systems can often perturb the magnetic properties of the system. The magnet poles are mounted inside the UHV chamber, while the coils are mounted externally on a yoke, which connects each pair of magnet poles via custom vacuum feedthroughs. A magnetic field of 2800 Oe can be obtained for a gap width of $15 \mathrm{~mm}$. The capabilities of the instrument are demonstrated by an investigation of ultrathin $\mathrm{Fe}$ films grown on $\mathrm{Mo}(110)$.

The SMOKE system is part of a larger UHV system, which also comprises a room-temperature STM, low energy electron diffraction (LEED), and Auger electron spectroscopy (AES) facilities and a small chamber for thin film deposition equipped with a fast-entry loadlock. The typical base pressure achieved in this system is in the low $10^{-10}$ Torr. Figure 1(a) shows a cross-sectional schematic of the electromagnet assembly mounted on the UHV chamber. The pole tips are located inside the UHV chamber, while the rest of the assembly is mounted outside. The poles are machined from UHV compatible Permenorm 5000 H2 alloy (Vacuumschmelze $\mathrm{GmbH}$ ) and are tapered to achieve higher fields and to prevent blocking the laser from the sample. ${ }^{2}$ They are coupled to an external core (made from low carbon steel) via customized vacuum feedthroughs machined from the same alloy [Fig. 1(b)]. The feedthroughs are mounted on linear translators so that the gap between the poles can be adjusted. The maximum magnetic field achieved can therefore be increased for smaller samples. This arrangement also allows the poles to be withdrawn during sample transfer. An externally mounted yoke connects the core pieces and closes the magnetic field path. There are slots in the core pieces so that they can slide along the yoke when the poles are moved. The magnetic field is produced by four external coils, each made from 400 turns of $2 \mathrm{~mm}$ diameter insulated copper wire. A switch, containing a pair of magnetomechanical relays is inserted between the bipolar power supply and the coils so that the magnetic field can be reversed. The magnetic field in the gap was calibrated versus current using a Hirst GM 05 Gaussmeter. A magnetic field of $\sim 2800$ Oe can be obtained for a $15 \mathrm{~mm}$ gap by driving a current of $10 \mathrm{~A}$ current through the coils. The sample is mounted on a copper block located in the gap, which is attached to a liquid nitrogen cooled cold finger. The copper block is also fitted with a resistive heater made from high resistivity Nichrome wire and a K-type thermocouple. These are linked to a PID temperature controller so that the temperature of the block can be stabilized at any temperature between 85 and $500 \mathrm{~K}$. The accuracy of the temperature measurements is $\pm 1.5 \mathrm{~K}$. The sample sits in an insert screwed into the block with a fine pitch thread to provide the azimuthal rotation for the sample.

A block diagram of the SMOKE setup is shown in Fig. 2. The light source is a diode laser with a wavelength of $\lambda$ $=685 \pm 10 \mathrm{~nm}$. Two sheet polarizers with an extinction ratio of $10^{-4}$ are used to define the polarization of the incident light ( $p$-polarized light is used) and as the analyzer in front of the detector. The light intensity is detected by a photodiode with an integrated amplifier. A convex lens is placed in front of the photodiode to focus the beam onto the photodiode's active area. To reduce the unwanted depolarizing effect due to the birefringence of the UHV viewports, a retarder is placed in front of the analyzing polarizer. The analyzing polarizer is set at an angle of $2^{\circ}-3^{\circ}$ from the 

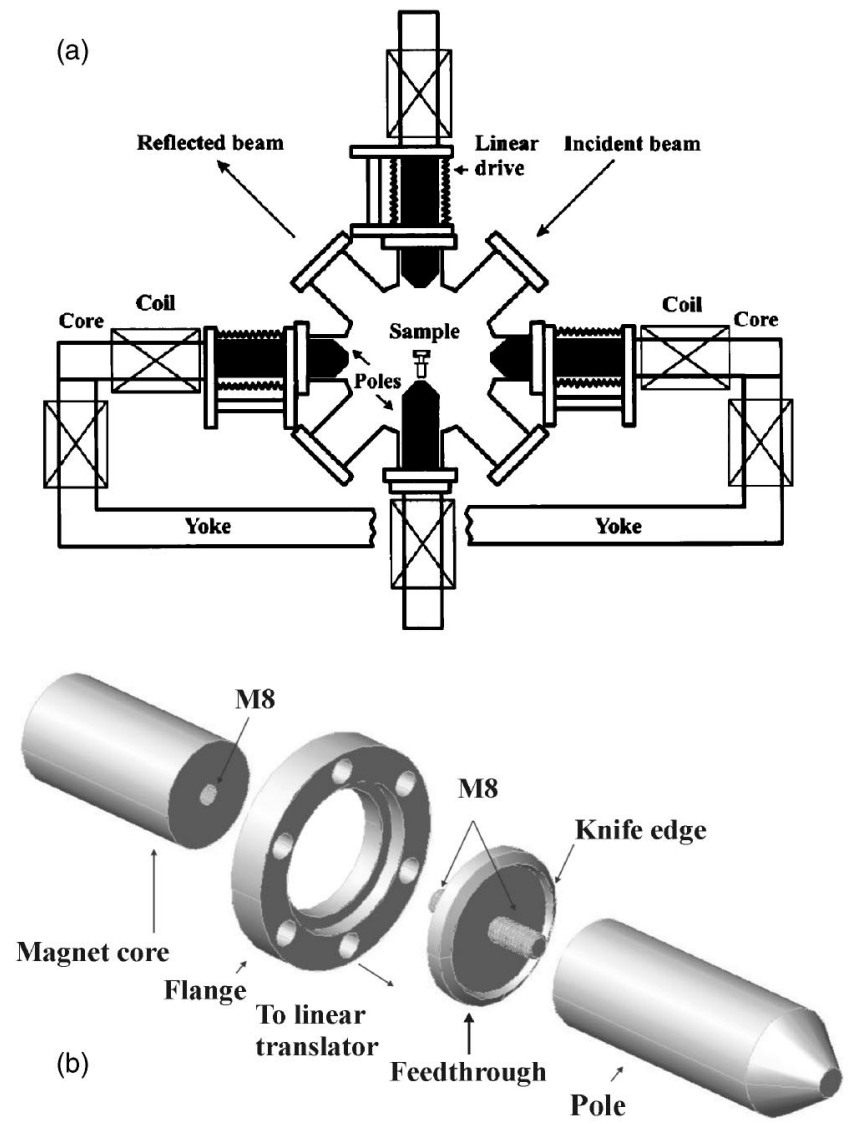

FIG. 1. (a) Schematic cross section of the SMOKE chamber with the electromagnet assembly. The magnet pole pairs are inserted in the UHV chamber on linear translators. The coils are mounted externally on a yoke, which is swapped between the pole pairs depending on whether in-plane or out-ofplane measurements are performed; (b) schematic of the magnet pole, vacuum feedthrough, and core. The external yoke is bolted to the core to close the magnetic field path.

$p$-axis, where the maximum contrast is achieved. ${ }^{1}$ To eliminate stray light from external sources and to enhance the signal-to-noise ratio, the laser intensity is internally modulated at a frequency of about $1.5 \mathrm{kHz}$. This modulated signal is provided to the reference input of a lock-in amplifier, while the photodetector output signal is applied to the input of the lock-in amplifier. The filtered output is digitized by a digital multimeter, which is connected to a computer via a

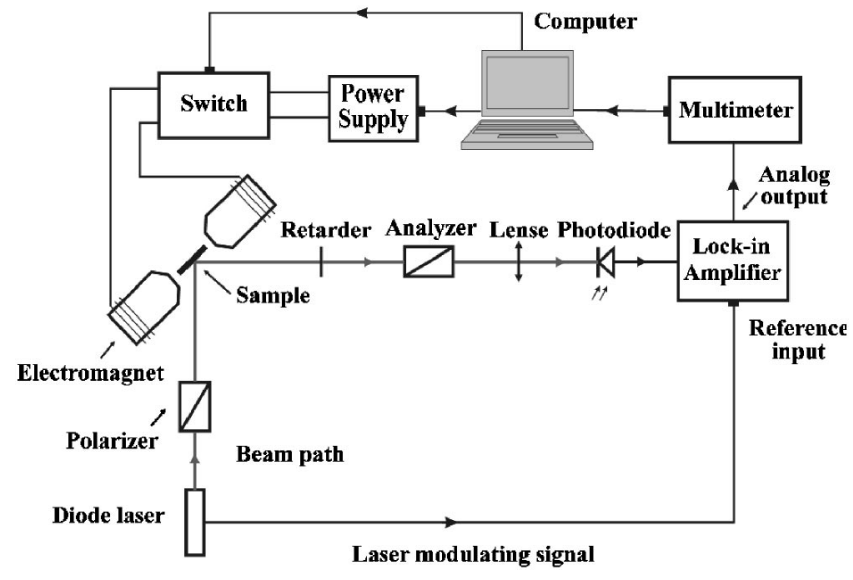

FIG. 2. Block diagram of the SMOKE experiment including optical components.

GPIB interface. To speed up the measurements, the current step applied to the electromagnet is set smaller in the region of magnetic reversal and larger at the ends of the hysteresis loop. It takes from 10 to $60 \mathrm{~s}$ to record a single hysteresis loop. Prior to the measurement, the magnetic field is repeatedly reversed to cycle the film around the hysteresis loop and after several reversals the tips of the loop become reproducible. To improve the signal-to-noise ratio, the hysteresis loop can be measured multiple times and then averaged.

Aside from birefringence, the Voigt effect is another unsought phenomenon introduced by the UHV viewports. This occurs when the viewports are subjected to stray magnetic fields from the electromagnet. The Voigt effect describes the change in the light's ellipticity and the rotation of its plane of polarization as it propagates through a medium placed in a magnetic field orthogonal to the wave vector. In an isotropic medium such as glass, the lowest order term of Voigt effect is quadratic in the applied magnetic field. SMOKE hard axis hysteresis loops distorted by the Voigt effect from UHV windows have been observed in a number of SMOKE experiments. ${ }^{3,4}$ The influence of the Voigt effect is demonstrated in Fig. 3. The loop shown in Fig. 3(a) was recorded using the longitudinal configuration with a beam reflecting from a bulk Mo polycrystalline sample. Mo is a paramagnetic material with magnetic susceptibility $\chi=1.19 \times 10^{-4}$ and therefore no changes in the light intensity should be
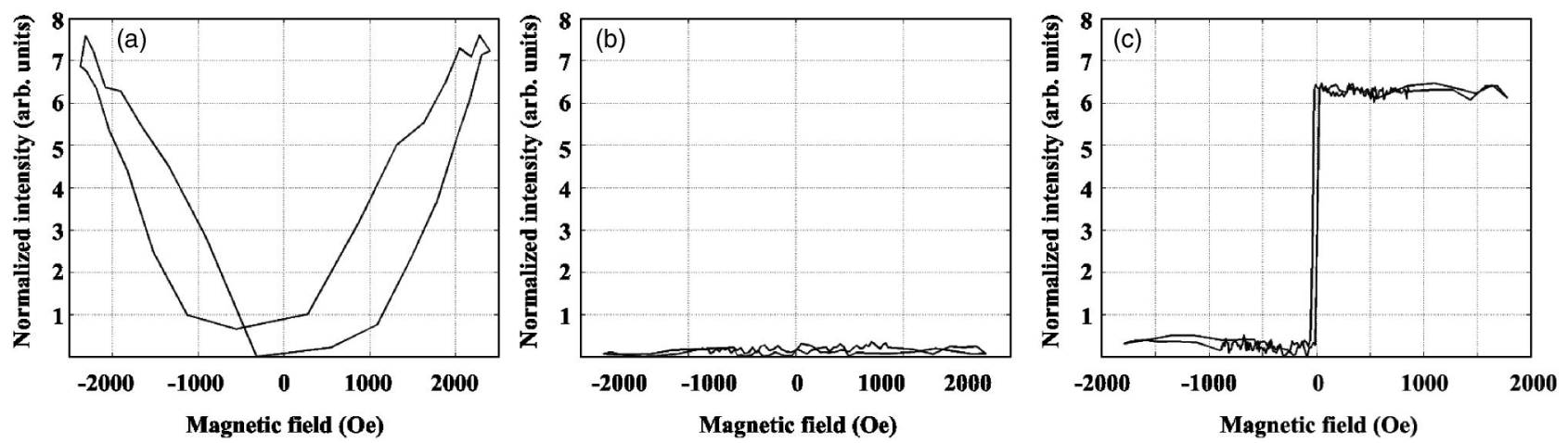

FIG. 3. Intensity of light transmitted through the analyzer (normalized to the intensity at $H=0$ ) vs longitudinal magnetic field for: (a) a polycrystalline molybdenum disk. The changes in the output intensity are proportional to $H^{2}$ and result from the Voigt effect due to the UHV windows; (b) same as (a) but with magnetic shields placed over the viewports; (c) easy axis MOKE loop produced by a $6 \mathrm{ML} \mathrm{Fe} / \mathrm{Mo}(110)$ film when the experiment is performed with the shielded viewports. 


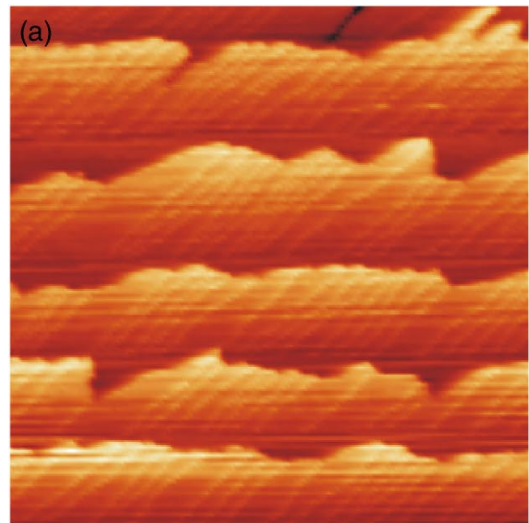

(b)
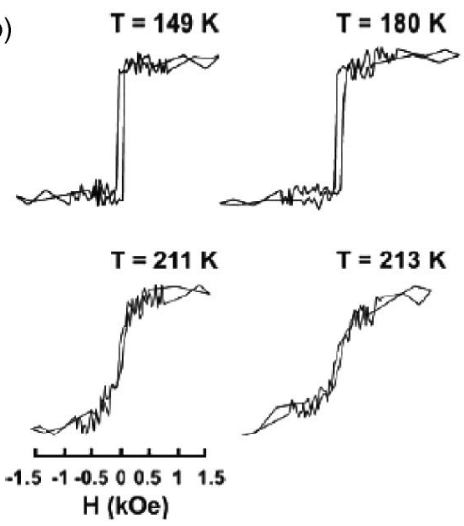

$\mathrm{T}=209 \mathrm{~K}$
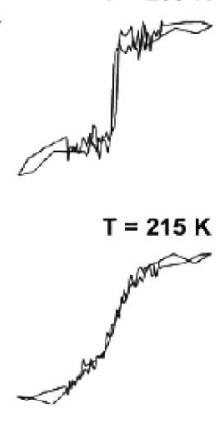

FIG. 4. (a) $250 \times 250 \mathrm{~mm}^{2} \mathrm{STM}$ image of a $1.85 \mathrm{ML} \mathrm{Fe} / \mathrm{Mo}(110)$ film grown at $510 \mathrm{~K}$; (b) Hysteresis loops measured at indicated temperatures for a $1.95 \mathrm{ML} \mathrm{Fe} / \mathrm{Mo}(110)$ film grown at $510 \mathrm{~K}$. observed in the whole range of the magnetic field accessible in our setup. However, it is clear that there are changes in the output signal that are proportional to $H^{2}$. The loop shown in Fig. 3(b) was obtained from the same polycrystalline Mo sample, but with Permalloy tubes slotted over the viewports to shield the glass from the stray magnetic field produced by the electromagnet. These magnetic shields eliminate the unwanted magneto-optical effect. Figure 3(c) shows a loop measured on a $6 \mathrm{ML} \mathrm{Fe/Mo(110)} \mathrm{film,} \mathrm{which} \mathrm{demonstrates}$ that the Voigt effect on the UHV windows is comparable in magnitude to the Kerr intensity obtained from an ultrathin $\mathrm{Fe}$ film.

To demonstrate the capabilities of this instrument, we have studied magnetic behavior of a nearly complete $2 \mathrm{ML}$ Fe film deposited on a Mo(110) surface at $510 \mathrm{~K}$. A STM image of this film is shown in Fig. 4(a). The growth proceeds by the step-flow mechanism producing 2 ML thick Fe stripes aligned along the atomic steps. ${ }^{5}$ The stripes cover the entire surface of the substrate forming a structure that can be considered as a good experimental approximation of a two- dimensional ferromagnetic system. Longitudinal hysteresis loops displayed in Fig. 4(b) were measured with the magnetic field along the [001] crystallographic direction, which is the magnetic easy axis for $\mathrm{Fe} / \mathrm{Mo}(110) .{ }^{6}$ Well below the Curie temperature the loops are square with the full remanence, which decreases with increasing temperature and finally disappears at the Curie point.

This work was supported by Science Foundation-Ireland under Contract No. 00/PI.1/C042.

${ }^{1}$ E. R. Moog and S. D. Bader, Superlattices Microstruct. 1, 543 (1985); S. D. Bader, E. R. Moog, and P. Grünberg, J. Magn. Magn. Mater. 53, L295 (1986)

${ }^{2}$ C. Liu, E. R. Moog, and S. D. Bader, Phys. Rev. Lett. 60, 2422 (1988).

${ }^{3}$ C. Liu and S. D. Bader, J. Vac. Sci. Technol. A 8, 2727 (1990).

${ }^{4}$ D. Sanders, A. Enders, C. Schmidthals, D. Reuter, and J. Kirshner, Surf. Sci. 402-404, 351 (1998).

${ }^{5}$ S. Murphy, D. Mac Mathúna, G. Mariotto, and I. V. Shvets, Phys. Rev. B 66, 195417 (2002).

${ }^{6}$ V. Usov, S. Murphy, and I. V. Shvets, J. Magn. Magn. Mater. 283, 357 (2004). 